\section{Anabolic steroids for rehabilitation after hip fracture in older people}

This is the abstract of a Cochrane Review published in the Cochrane Database of Systematic Reviews (CDSR) 2014, issue 10. art. no.: CD008887. DOI: 10.1002/14651858.CD008887.pub2

\author{
Vaqas Farooqi, Maayken E. L. van den Berg, \\ Ian D. Cameron, Maria Crotty
}

The independent commentary was written by Mauricio de Miranda Ventura

\section{ABSTRACT}

BACKGROUND: Hip fracture occurs predominantly in older people, many of whom are frail and undernourished. After hip fracture surgery and rehabilitation, most patients experience a decline in mobility and function. Anabolic steroids, the synthetic derivatives of the male hormone testosterone, have been used in combination with exercise to improve muscle mass and strength in athletes. They may have similar effects in older people who are recovering from hip fracture.

OBJECTIVES: To examine the effects (primarily in terms of functional outcome and adverse events) of anabolic steroids after surgical treatment of hip fracture in older people.

METHODS:

Search methods: We searched the Cochrane Bone, Joint and Muscle Trauma Group Specialized Register (10 September 2013), the Cochrane Central Register of Controlled Trials (CENTRAL) (The Cochrane Library, 2013 Issue 8), MEDLINE (1946 to August Week 4 2013), EMBASE (1974 to 2013 Week 36), trial registers, conference proceedings, and reference lists of relevant articles. The search was run in September 2013.

Selection criteria: Randomized controlled trials of anabolic steroids given after hip fracture surgery, in inpatient or outpatient settings, to improve physical functioning in older patients with hip fracture.

Data collection and analysis: Two review authors independently selected trials (based on predefined inclusion criteria), extracted data and assessed each study's risk of bias. A third review author moderated disagreements. Only very limited pooling of data was possible. The primary outcomes were function (for example, independence in mobility and activities of daily living) and adverse events, including mortality.

MAIN RESULTS: We screened 1290 records and found only three trials involving 154 female participants, all of whom were aged above 65 years and had had hip fracture surgery. All studies had methodological shortcomings that placed them at high or unclear risk of bias. Because of this high risk of bias, imprecise results and likelihood of publication bias, we judged the quality of the evidence for all primary outcomes to be very low.

These trials tested two comparisons. One trial had three groups and contributed data to both comparisons. None of the trials reported on patient acceptability of the intervention.

Two very different trials compared anabolic steroid versus control (no anabolic steroid or placebo). One trial compared anabolic steroid injections (given weekly until discharge from hospital or four weeks, whichever came first) versus placebo injections in 29 "frail elderly females". This found very low quality evidence of little difference between the two groups in the numbers discharged to a higher level of care or dead (one person in the control group died) (8/15 versus $10 / 14$; risk ratio
(RR) $0.75,95 \%$ confidence interval $(\mathrm{Cl}) 0.42$ to $1.33 ; \mathrm{P}=0.32$ ), time to independent mobilization or individual adverse events. The second trial compared anabolic steroid injections (every three weeks for six months) and daily protein supplementation versus daily protein supplementation alone in 40 "lean elderly women" who were followed up for one year after surgery. This trial provided very low quality evidence that anabolic steroid may result in less dependency, assessed in terms of being either dependent in at least two functions or dead (one person in the control group died) at six and 12 months, but the result was also compatible with no difference or an increase in dependency (dependent in at least two levels of function or dead at 12 months: $1 / 17$ versus 5/19; RR $0.22,95 \% \mathrm{Cl} 0.03$ to $1.73 ; \mathrm{P}=0.15)$. The trial found no evidence of between-group differences in individual adverse events.

Two trials compared anabolic steroids combined with another nutritional intervention ('steroid plus') versus control (no 'steroid plus'). One trial compared anabolic steroid injections every three weeks for 12 months in combination with daily supplement of vitamin D and calcium versus calcium only in 63 women who were living independently at home. The other trial compared anabolic steroid injections every three weeks for six months and daily protein supplementation versus control in 40 "lean elderly women". Both trials found some evidence of better function in the steroid plus group. One trial reported greater independence, higher Harris hip scores and gait speeds in the steroid plus group at 12 months. The second trial found fewer participants in the anabolic steroid group were either dependent in at least two functions, including bathing, or dead at six and 12 months (one person in the control group died) (1/17 versus $7 / 18$; RR $0.15,95 \% \mathrm{Cl} 0.02$ to 1.10 ; $P=0.06)$. Pooled mortality data ( $2 / 51$ versus $3 / 51$ ) from the two trials showed no evidence of a difference between the two groups at one year. Similarly, there was no evidence of between-group differences in individual adverse events. Three participants in the steroid group of one trial reported side effects of hoarseness and increased facial hair. The other trial reported better quality of life in the steroid plus group. AUTHORS' CONCLUSIONS: The available evidence is insufficient to draw conclusions on the effects, primarily in terms of functional outcome and adverse events, of anabolic steroids, either separately or in combination with nutritional supplements, after surgical treatment of hip fracture in older people. Given that the available data points to the potential for more promising outcomes with a combined anabolic steroid and nutritional supplement intervention, we suggest that future research should focus on evaluating this combination.

The full-text of this review (English), the abstract (English and French) and a plain language summary (for patients and consumers, in English and French) are available from: http://onlinelibrary.wiley.com/ doi/10.1002/14651858.CD008887.pub2/full

\section{REFERENCE}

1. Faroogi V, van den Berg ME, Cameron ID, Crotty M. Anabolic steroids for rehabilitation after hip fracture in older people. Cochrane Database Syst Rev 2014;(10):CD008887.

\section{COMMENTS}

Sarcopenia is a trademark of aging that consists of progressive loss of muscle mass. When this is accompanied by functional impairment, thus compromising elderly people's independence and autonomy, the condition is known as frailty syndrome. This condition leads to decreased activity, mobility and bone mass and a predisposition towards falls, another 
geriatric syndrome of great importance. Both frailty syndrome and fall syndrome bring in their wake increased morbidity, mortality and compromised independence and autonomy among elderly people.

It might be thought that, after surgery on an elderly individual to correct a femoral fracture resulting from a fall, use of steroids alone for rehabilitation may be beneficial towards recovering the individual's mobility, independence and autonomy. However, this would ignore the whole process and physiopathological condition that led to this event.

Fundamentally, the history of patients who have fallen needs to be known. Were they previously independent and autonomous? Do they have any chronic degenerative disease (hypertension, diabetes mellitus, heart failure or stroke sequelae)? What were their cognition levels prior to the fall (did they present dementia?)? Within this age group, patients have widely varying states of functionality, which is well known to interfere with making prognoses.

One important issue in evaluating elderly individuals' recovery concerns their functionality, particularly in comparison with their previous condition. The main objective of any procedure and treatment for patients in this age group is to maintain their independence and autonomy.

In analyzing sarcopenia in elderly people, coupled with frailty and fall syndromes, this process needs to be understood as part of a set of factors. Malnutrition per se does not have as important a role in relation to the prevalence of sarcopenia as low protein intake does. Other factors involved include physical inactivity (which accentuates the loss of muscle mass), neuroendocrine deregulation (for which the most classic examples are glucose intolerance and hypothyroidism) and immune dysfunction (for example, increased levels of interleukins 1 and 6, and of tumor necrosis factor, and decreased cellular immunity). Given this complexity of events that culminate in sarcopenia among elderly people, decreased testosterone levels in these patients are only rarely seen. It has already been shown that hormone supplementation does not meet the expectations for improvement of muscle mass and functional performance, especially when this is done alone, without taking the other factors into consideration. It is unsurprising that when physical activity is added to administration of steroids, the results tend to be a little better. However, these results are not so significantly better as to justify use of steroids. There have not been any reports of improved muscle strength in frail elderly individuals through calcium replacement. Until not long ago, it was believed that vitamin D replacement at high doses could reduce the loss of muscle strength, thus reducing the risk of falls and fractures. However, even this has been questioned recently.

Lastly, the issue of tolerability was undervalued in these studies, given that diseases strongly associated with steroid replacement, such as atherosclerotic vascular diseases and neoplastic diseases (liver, testes and prostate), were not even mentioned. The short durations of these studies will clearly have interfered with such associations, but some kind of assessment in this regard, so as to ensure that the therapy was tolerable, should have been conducted.

Therefore, it is not surprising that the results from this systematic review do not recommend steroid replacement for improving muscle strength in elderly individuals subsequent to operative femoral fracture repair. The reality of frail elderly individuals who fall is much more complex than this.

Mauricio Miranda Ventura, MD, MSc. Technical Director of the Geriatrics Service of Hospital do Servidor Público Estadual, São Paulo; Head of the Department of Geriatrics and Gerontology, Associação Paulista de Medicina, São Paulo; Secretary-General of the Brazilian Society of Geriatrics and Gerontology, São Paulo section; and Coordinator of Internships on Elderly's People Health at Universidade Cidade de São Paulo, São Paulo. 